\title{
Los Hábitos de Lectura y Escritura en los Estudiantes de Educación Primaria: un Análisis Dentro y Fuera de la Escuela
}

\author{
Celia Moreno-Morilla \\ FERnANDo GuZMÁN-SimÓn \\ EDUARDO GARCÍA-JiMÉNEZ \\ Universidad de Sevilla
}

Recibido: 30 abril 2017 / Aceptado: 21 julio 2017

ISSN: 1697-7467

\begin{abstract}
RESUMEN: Los cambios de los eventos letrados en Educación Primaria a través de las TIC y la cultura popular han motivado la aparición de nuevas comunidades, dominios y discursos escritos. Dichas comunidades han desarrollado eventos vernáculos que han quedado al margen de estudios precedentes. Nuestra investigación aborda los tipos, frecuencia y dominios donde se desarrollan los eventos letrados de los estudiantes de Educación Primaria. Del mismo modo, ha determinado los distintos perfiles alfabetizadores y la diversidad de estos según el Índice Socioeconómico y Cultural. La investigación se realizó a través de análisis descriptivo (análisis estadístico univariantes) y correlacional-predictivo (análisis de clúster bietápico). La muestra estuvo compuesta por 1540 escolares de $2^{\circ}$ y $3^{\text {er }}$ ciclo de EP y la información fue recogida mediante un autoinforme. Los resultados obtenidos evidencian una heterogeneidad de los eventos letrados entre las distintas edades y según el ISC de los centros. En cambio, muestra la homogeneidad de los eventos letrados desarrollados en el contexto escolar. En conclusión, esta investigación viene a reforzar la tesis que defiende que los factores sociales poseen un mayor peso que las diferencias individuales en el desarrollo de la alfabetización en EP.

Palabras clave: Educación Primaria, nivel socioeconómico, lectura, escritura, relación familia-escuela
\end{abstract}

Primary education students' reading and writing habits: an analysis in and out of school.

ABSTRACT: The changes of literacy events in Primary Education both through ICT and social culture have motivated the emergence of new communities, domains and written speech. These communities have developed new practices that have been excluded from previous studies. Our research has addressed the types, frequencies and domains on which literacy practices of PE students are developed. Similarly, our work has analysed the different literacy profiles and the diversity of these according to the index of the student's socioeconomic status. This research carries out through descriptive and correlational-predictive analysis (Two-Step Cluster Analysis). The sample consisted of 1540 students of 2nd and 3rd cycle of PE and the information was collected through a self-reporting survey. The results show some differences on the practices between the different ages and the schools' socioeconomic status. On the other hand, it shows similar practices developed in the school context. In conclusion, this research reinforces the hypothesis that social factors have a greater weight than individual differences in the development of literacy in PE.

Keywords: Primary education, socioeconomic level, reading, writing, family school relationship

Esta investigación fue financiada por el Ministerio de Educación, Cultura y Deporte de España (FPU-14/05336). 


\section{INTRODUCCIÓN}

Una revisión de los estudios realizados sobre la lectura y la escritura en España en la última década arroja cierta descompensación entre la preocupación educativa por la comprensión lectora (a través de los informes internacionales como el Informe PIRLS de 2011) y el conocimiento de los hábitos lectores en etapas iniciales de escolarización como la Educación Primaria (en adelante, EP). Los pobres resultados obtenidos en comprensión lectora no se corresponden con el panorama descrito por el Observatorio de la Lectura y el Libro en España (cfr. Encuestas de Hábitos y prácticas culturales 2014-15 [MEC, 2015]), la Federación de Editores de España (Millán, 2017) y los estudios del Centro de Investigaciones Sociológicas (CIS, febrero 2016; septiembre 2016). En particular, el Observatorio de la Lectura y el Libro dibuja un panorama donde los estudiantes tienen unos excelentes hábitos lectores y en el que cabe destacar que el 84,6\% leen en su tiempo libre y no exclusivamente por sus estudios. Sin embargo, esa misma fuente recoge que la lectura se realiza exclusivamente en los centros educativos y en las bibliotecas y no se contempla otra forma de lectura y escritura que no sea la impresa. Las limitaciones de estas investigaciones (una población mayor de catorce años y el predominio de la lectura literaria impresa) impiden un acercamiento más global a los procesos alfabetizadores de los estudiantes. En general, los estudios sobre las actividades de los escolares con la lengua escrita (eventos letrados) y dónde se realizan (dominio) se han desarrollado escasamente en España en comparación con otros países europeos (cfr. Formby, 2014). Un estudio pionero en el análisis de los hábitos lecto-escritores en nuestro país es el realizado por Gómez-Soto (1999). Este trabajo analiza dichos hábitos, a partir de encuestas realizadas por el INE y el CIS desde el año 1964 hasta 1997, y los pone en relación con variables de tipo personal, contextual y cultural (sexo, edad, nivel de estudios, ISC, hábitos de consumo, uso de bibliotecas, etc.).

Los nuevos contextos alfabetizadores a través de las Tecnologías de la Información y la Comunicación (en adelante, TIC) y la cultura popular nos invitan a cuestionar los resultados de dichos estudios y analizar en profundidad en qué medida las TIC están cambiando los hábitos lectores y escritores de los alumnos ${ }^{1}$ en una etapa como la EP.

\section{Marco Teórico}

\subsection{Los cambios de hábitos alfabetizadores en EP}

La mayoría de las investigaciones sobre los hábitos lectores en EP no refleja la heterogeneidad de la alfabetización en la actualidad. Los datos obtenidos hasta ahora no han hecho visible otras formas de lectura y escritura diferentes a las desarrolladas en la escuela y han omitido aquellas que tienen lugar en dominios tan distintos como la familia, los amigos u otras comunidades (Duursma, Meijer \& De Bot, 2017; Hull \& Shultz, 2002). Los eventos letrados, considerados como actividades donde la alfabetización tiene algún tipo de papel social (Heath, 1983), relacionan la lectura y la escritura no sólo con el dominio escolar, sino

\footnotetext{
${ }^{1}$ Los términos alumnos, estudiantes y otros similares presentan un género gramatical no marcado.
} 
con cualquier actividad diaria donde el código escrito está presente en los estudiantes de EP, como en el dominio hogar, en la escuela y en el mercado (Neuman \& Celano, 2001). Para comprender la heterogeneidad de la alfabetización de nuestros días, debemos distinguir los conceptos dominio y espacio. El primero es el lugar donde un evento letrado es creado y repetido en situaciones análogas; el segundo, es el emplazamiento donde el evento letrado ha sido desarrollado. El ejemplo más claro sobre la diferencia entre dominio y espacio es la tarea de lectura que el maestro envía a sus alumnos para que se realice en casa, donde la lectura pertenece al dominio escuela, pero es realizada en el dominio hogar (Pahl \& Rowsell, 2012).

Diversos estudios han destacado el papel del "dominio" como eje sobre el que se construye los valores sociales de los eventos letrados (Barton \& Hamilton, 1998). En líneas generales, los eventos letrados se desarrollan tanto en dominios locales (p.e. en un espacio físico) como globales (p.e. mediante las TIC); aunque en realidad toda alfabetización es local (Brandt \& Clinton, 2002). En definitiva, las TIC han cambiado la manera en que se desarrolla la comunicación local (Lillis, 2013), tal y como podemos observar en cualquier comunidad compuesta por estudiantes de EP (Maybin, 2006). Dichos escolares combinan a diario sistemas de alfabetización digital e impresa en diversos dominios locales (Kalantzis $\&$ Cope, 2000).

Una consecuencia de lo anterior es que el estudio de la alfabetización debería atender a la heterogeneidad de dominios sociales y eventos letrados que desarrollan los niños durante su escolarización en etapas iniciales como la EP. Esto permitiría atender tanto a los eventos letrados visibles, como a aquellos otros "invisibles" o vernáculos que se desarrollan fuera del dominio escuela (Pahl \& Allan, 2011). Con este fin, en la Tabla 1 hemos clasificado los eventos letrados en los que participan los escolares en cuatro dimensiones.

Tabla 1. Dimensiones de los eventos letrados en EP

\begin{tabular}{|c|l|}
\hline DIMENSIÓN & \multicolumn{1}{c|}{ DESCRIPCIÓN } \\
\hline $\begin{array}{c}\text { Alfabetización } \\
\text { personal }\end{array}$ & $\begin{array}{l}\text { Eventos letrados que se desarrollan en diferentes espacios y son adquiridos } \\
\text { como aprendizajes no-formales e informales. }\end{array}$ \\
\hline $\begin{array}{c}\text { Producción y consumo } \\
\text { cultural }\end{array}$ & $\begin{array}{l}\text { Eventos letrados que se desarrollan en diferentes espacios y están } \\
\text { relacionados con la compra y venta de productos letrados. }\end{array}$ \\
\hline Cultura de la memoria & $\begin{array}{l}\text { Eventos letrados que se desarrollan en diferentes espacios y se relacionan } \\
\text { con el uso de bibliotecas públicas y privadas. }\end{array}$ \\
\hline $\begin{array}{c}\text { Cultura de la } \\
\text { instrucción }\end{array}$ & $\begin{array}{l}\text { Eventos letrados que se desarrollan en diferentes espacios y son adquiridos } \\
\text { como aprendizajes formales en una institución educativa. }\end{array}$ \\
\hline
\end{tabular}

Fuente: Elaboración propia

\subsection{Las nuevas tecnologías y el cambio en la alfabetización}

En el contexto social actual en el que se desenvuelven los estudiantes de EP el discurso escrito está omnipresente y no sólo está compuesto de textos escolares, sino también de videojuegos, redes sociales o mensajería instantánea (Barton \& Papen, 2010). Estas prácticas no 
escolares o vernáculas de alfabetización son en su mayoría discursos multimodales (Lancaster, 2003; Pahl, 2007), desarrollados a partir de un estilo de aprendizaje eminentemente no-formal o informal (Mocker \& Spear, 1982), y tienen lugar en un contexto de comunicación on-line (Barton \& Lee, 2012). En consecuencia, se trata de procesos vernáculos de alfabetización que interaccionan con aquellos otros aprendidos en la escuela (Compton-Lilly \& Green, 2011).

La utilización de distintos dispositivos digitales en dominios escolares y familiares ha transformado los procesos de enseñanza-aprendizaje en las escuelas de EP (Dagenais, Toohey, Fox, \& Singh, 2016; Hashemi, 2017; Lotherington, 2017). Esta circunstancia ha inaugurado una heterogénea línea de investigación en distintos contextos educativos en las últimas décadas (Goodfellow, 2011). La pluralidad de alfabetizaciones que desarrollan los niños en distintos dominios ha requerido la configuración del concepto alfabetización múltiple (New London Group, 1996; Cope \& Kalantzis, 2009). Dicha alfabetización alude a los conocimientos y usos de una gramática multimodal (Kress, 2003) en diversos dominios, especialmente los familiares y personales (Williams, 2009).

Esta situación ha reducido la capacidad de las escuelas para controlar el proceso de alfabetización de los estudiantes de EP (Davies \& Merchant, 2009), mientras que ha crecido la influencia de la cultura popular (como los juegos de ordenador, la música popular o la televisión). Asimismo, las TIC han adquirido un papel protagonista en el desarrollo de la alfabetización en el dominio hogar (Gregory \& Williams, 2000), a través de numerosos aprendizajes informales y no formales (Rowsell \& Pahl, 2007). Estos cambios sociales y sus consecuencias en los procesos de alfabetización requieren una mejor comprensión, tanto de los eventos letrados como de los dominios en los que se desarrollan.

\subsection{Las prácticas de alfabetización y los indicadores socioculturales de los centros escolares}

La relación entre el ISC (Índice Socioeconómico y Cultural) de los centros escolares y el tipo y grado de alfabetización de los escolares en EP ha sido demostrada en numerosos estudios precedentes (Caro, McDonald \& Willms, 2009; Desert, Preaux \& Jund, 2009; Van Steensel, 2006, entre otros). El interés por conocer los perfiles actuales de los lectores y escritores en EP dentro y fuera de la escuela nos lleva a preguntarnos si existe una relación entre los distintos ISC de los centros y el tipo de alfabetización que desarrollan en edad escolar (Compton-Lilly, 2009; Purcell-Gates, 1995; 1996).

En el marco de los estudios sobre el ISC y el rendimiento se ha destacado la necesidad de analizar la alfabetización de los niños prestando atención a los eventos letrados y a su relación con el entorno familiar y su ISE (nivel socieconómico) (Dixon \& Wu, 2014; Gil, 2013). Hasta hoy, las investigaciones habían relacionado el ISE con el rendimiento escolar (Bourdieu \& Passeron, 1990) pero sin profundizar en los tipos de eventos letrados que diferencian a los alumnos de EP de familias con diferente ISE (cfr. Van Steensel, 2006).

\section{Nuestra inVESTIGACIÓN}

Una de las líneas de investigación llevadas a cabo en las últimas décadas ha tenido como foco de atención la descripción de los eventos letrados de los estudiantes en EP. La 
finalidad de un análisis cuantitativo de los hábitos lectores y escritores de los estudiantes de EP permitirá la búsqueda de la relación predictiva entre los tipos y frecuencias de eventos alfabetizadores dentro y fuera de escuela según el ISE. Esto reportará diversos resultados sobre el comportamiento de la alfabetización en EP. Este artículo se centra en la descripción en términos extensivos de los hábitos de lectura y escritura presentes en los dominios escuela y hogar. No obstante, el trabajo en el que se basa forma parte de un diseño de investigación mixto, en el que se abordan las relaciones en el proceso alfabetizador existente entre las familias y la escuela. En dicho diseño se incorporan 30 estudios de casos anidados, siguiendo la propuesta de Yin (2014), que completan con una metodología cualitativa el trabajo cuantitativo aquí presentado. En el desarrollo de la aproximación cualitativa se toman en consideración los trabajos de Sarland (2003), Tusting y Barton (2005) y Pahl y Rowsell (2012).

Nuestro acercamiento cuantitativo pretende construir un claro perfil generalizador de las percepciones de los niños en EP sobre la alfabetización a través de distintos medios y situaciones, sean en un contexto escolar o familiar, lectura impresa o digital, prácticas institucionales o vernáculas y tipos de aprendizaje (formal, informal o no formal). Con este fin nos hemos propuesto los siguientes objetivos:

a. Describir las prácticas y eventos letrados de los alumnos en EP.

b. Identificar los perfiles lectores y escritores de los alumnos de EP.

\section{Metodología}

Con el fin de dar respuesta a los objetivos planteados en la investigación se emplean diferentes metodologías anotadas en la Tabla 2 en un diseño ex post facto.

Tabla 2. Resumen Metodológico

\begin{tabular}{|l|c|c|c|}
\hline \multicolumn{1}{|c|}{ Objetivo } & Método & Instrumento & Tipo de análisis \\
\hline $\begin{array}{l}\text { Describir las prácticas y eventos letrados de } \\
\text { los alumnos en EP (Barton, 2007; Hamilton, } \\
\text { 2000). }\end{array}$ & Descriptivo & Modalidad & $\begin{array}{c}\text { Análisis basado } \\
\text { en estadísticos } \\
\text { univariantes }\end{array}$ \\
\cline { 1 - 2 } $\begin{array}{l}\text { Identificar los perfiles lectores y escritores } \\
\text { de los alumnos de EP (Pahl \& Rowsell, } \\
\text { 2005; Cope, 1999). }\end{array}$ & $\begin{array}{c}\text { Correlacional- } \\
\text { predictivo }\end{array}$ & $\begin{array}{c}\text { autoinforme } \\
\text { Análisis de clúster } \\
\text { bietápico }\end{array}$ \\
\hline
\end{tabular}

Fuente: Elaboración propia 


\subsection{Muestra}

En este estudio participaron veinte centros educativos de la provincia de Sevilla, que representan los diversos niveles de ISC (Tabla 3). En ellos se recogió información sobre las prácticas alfabetizadoras de 1540 escolares de segundo y tercer ciclo de EP, sobre un total de1843 matriculados.

Tabla 3. Distribución de los centros en función del ISC

\begin{tabular}{|c|c|c|c|c|c|c|c|c|c|c|c|}
\hline \multicolumn{2}{|c|}{$\begin{array}{l}\text { Alto } \\
(>0,36)\end{array}$} & \multicolumn{2}{|c|}{$\begin{array}{l}\text { Medio-Alto } \\
(-0,07 / 0,36)\end{array}$} & \multicolumn{2}{|c|}{$\begin{array}{c}\text { Medio } \\
(-0,32 /-0,07)\end{array}$} & \multicolumn{2}{|c|}{$\begin{array}{l}\text { Medio-Bajo } \\
(-0,62 / 0,32)\end{array}$} & \multicolumn{4}{|c|}{$\begin{array}{c}\text { Bajo } \\
(<-0,62)\end{array}$} \\
\hline $\mathrm{C} 1$ & 0,60 & $\mathrm{C} 3$ & 0,14 & C6 & $-0,12$ & C9 & $-0,47$ & $\mathrm{C} 12$ & $-0,70$ & $\mathrm{C} 16$ & $-0,91$ \\
\hline \multirow[t]{4}{*}{$\mathrm{C} 2$} & \multirow[t]{4}{*}{0,40} & $\mathrm{C} 4$ & 0,02 & C7 & $-0,26$ & $\mathrm{C} 10$ & $-0,59$ & C13 & $-0,66$ & $\mathrm{C} 17$ & $-0,72$ \\
\hline & & \multirow[t]{3}{*}{ C5 } & \multirow[t]{3}{*}{$-0,01$} & \multirow[t]{3}{*}{ C8 } & \multirow[t]{3}{*}{$-0,10$} & $\mathrm{C} 11$ & $-0,60$ & C14 & $-1,30$ & $\mathrm{C} 18$ & $-0,82$ \\
\hline & & & & & & & & \multirow[t]{2}{*}{$\mathrm{C} 15$} & \multirow[t]{2}{*}{$-0,90$} & $\mathrm{C} 19$ & $-1,02$ \\
\hline & & & & & & & & & & $\mathrm{C} 20$ & $-0,73$ \\
\hline
\end{tabular}

Fuente: Informes Pruebas Generales de Diagnóstico 2009 (Junta de Andalucía, Consejería de Educación)

\subsection{Procedimiento de recogida de información}

Para dar respuesta a los distintos objetivos propuestos fue aplicado un cuestionario a modo de autoinforme, que los estudiantes puntuaron según una escala ordenada en 6 valores. Este autoinforme, denominado "Las prácticas letradas en los alumnos de EP", disponible en https://es.surveymonkey.com/r/YB8FK52, persigue identificar las prácticas alfabetizadoras de los alumnos atendiendo principalmente a cuatro dimensiones: Alfabetización personal, Producción y consumo cultural, Cultura de la memoria y Cultura de la instrucción (Tabla 1). El autoinforme fue sometido a un estudio de validez y fiabilidad, obteniendo un Alpha de Cronbach de 0,95. El estudio de dicha fiabilidad por dimensiones arroja valores de Alpha en torno a 0,80, siendo la dimensión Cultura de la instrucción la que recoge el valor más alto $(0,91)$ y, Producción y consumo cultural, el más bajo $(0,76)$. Los resultados recogidos en la Tabla 4 sobre las medidas de desajuste (Stress, próximas a 0 ) y ajuste (D.AT. y C.C.T, próximas a la unidad) ratifican la adecuación de las dimensiones seleccionadas en el autoinforme. 
Tabla 4. Indicadores psicométricos del Autoinforme

\begin{tabular}{|c|c|c|c|c|c|c|c|}
\hline \multirow{2}{*}{ Dimensión } & Alpha de & \multicolumn{4}{|c|}{ Medidas de desajuste } & \multicolumn{2}{c|}{ Medidas de ajuste } \\
\cline { 5 - 9 } & Cronbach & $\mathrm{Sbn}^{2}$ & Stres I & Stres II & S-Stres & D.A.F & C.C.T \\
\hline $\begin{array}{c}\text { Alfabetización } \\
\text { personal }\end{array}$ & 0,84 & 0,002 & 0,147 & 0,320 & 0,031 & 0,978 & 0,989 \\
\hline $\begin{array}{c}\text { Producción y consumo } \\
\text { cultural }\end{array}$ & 0,76 & 0,038 & 0,196 & 0,354 & 0,029 & 0,961 & 0,980 \\
\hline Cultura de la memoria & 0,83 & 0,045 & 0,212 & 0,386 & 0,063 & 0,954 & 0,977 \\
\hline $\begin{array}{c}\text { Cultura de la } \\
\text { instrucción }\end{array}$ & 0,91 & 0,056 & 0,237 & 0,319 & 0,220 & 0,943 & 0,971 \\
\hline
\end{tabular}

Fuente: Elaboración propia

\subsection{Análisis de los datos}

El análisis de las respuestas al conjunto de los datos obtenidos del Autoinforme de los alumnos se ha realizado teniendo en cuenta diferentes métodos estadísticos. En primer lugar, la descripción de las prácticas alfabetizadoras se ha completado a partir de un análisis descriptivo (estadísticos univariantes). En segundo lugar, se realiza un análisis de clúster bietápico para determinar la importancia de las variables de contexto (centro y curso) y personales (sexo y edad) en la explicación de los diferentes perfiles alfabetizadores de los alumnos.

\section{Resultados}

\subsection{Resultados correspondientes al análisis descriptivo}

Los resultados obtenidos en relación a la dimensión Alfabetización personal presentan una amplia diversidad de dominios en los que suelen leer los escolares, siendo el ámbito escolar el más frecuente $\left(\overline{\mathrm{X}}=3,89\right.$; Coeficiente de Variación $\left.{ }^{3}=36,58\right)$ seguido del dominio familiar $(\bar{X}=3,80$; o $C V=37,02)$. En cambio, la biblioteca posee un papel testimonial en el desarrollo alfabetizador de los estudiantes $(\overline{\mathrm{X}}=1,43 ; \mathrm{CV}=42,48)$. La mayoría de los encuestados relacionan la actividad lectora con la adquisición de nuevos conocimientos $(\overline{\mathrm{X}}=4,12$; $\mathrm{CV}=30,34)$, siendo menos frecuente aquellos que identifican la lectura como actividad de disfrute u ocio $(\overline{\mathrm{X}}=3,38 ; C V=49,11)$. Los escolares muestran sus preferencias por la lectura de textos con imágenes o elementos audiovisuales $(\overline{\mathrm{X}}=3,72 ; \mathrm{CV}=44,35)$, de carácter narrativo principalmente $(\overline{\mathrm{X}}=3,73 ; \mathrm{CV}=44,50)$, seguido de la lectura de blogs y páginas web $(\overline{\mathrm{X}}=2,68$; $\mathrm{CV}=74,26)$. También, atribuyen a la escritura una funcionalidad escolar $(\overline{\mathrm{X}}=4,29 ; \mathrm{CV}=29,60)$ y tiene un carácter manuscrita $(\overline{\mathrm{X}}=4,72 ; \mathrm{CV}=16,95)$. En consecuencia, la escritura en medios digitales se reduce al desarrollo de prácticas alfabetizadoras personales, donde destaca por encima de todas el uso del WhatsApp $(\overline{\mathrm{X}}=3,45 ; \mathrm{CV}=56,81)$.

\footnotetext{
${ }^{2}$ Stress Bruto Normalizado

${ }^{3} \mathrm{CV}=$ Coeficiente de variación, resultante de dividir la desviación típica entre la media y multiplicar por cien el cociente resultante; a mayor CV mayor variabilidad en las respuestas respecto a la media.
} 
Figura 1. Dimensión Alfabetización personal

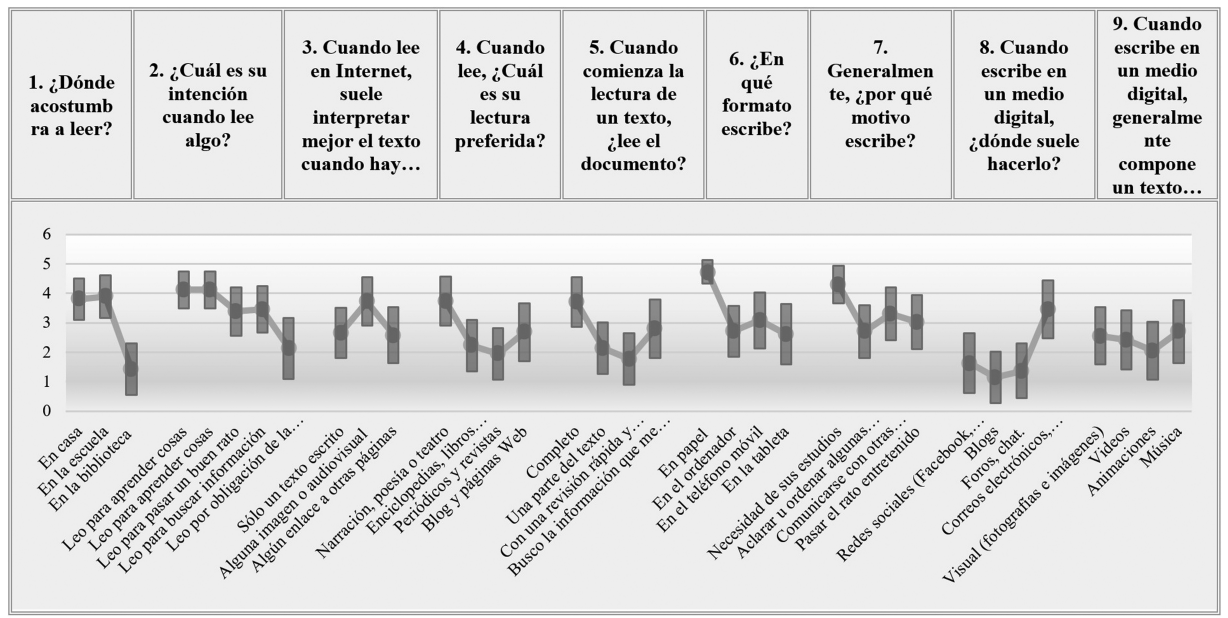

Fuente: Elaboración propia

El análisis de la dimensión Producción y consumo cultural de los estudiantes muestra un colectivo que suele comprar libros principalmente en librerías $(\overline{\mathrm{X}}=3,93 ; \mathrm{CV}=41,73)$ y centros comerciales $(\overline{\mathrm{X}}=2,34 ; \mathrm{CV}=84,62)$. En cambio, los estudiantes no están familiarizados con las compras online, obteniendo una media de 0,54 y un bajo coeficiente de variación $(23,33)$. En la mayoría de los casos, el motivo de sus compras puede explicarse desde las propias demandas que se originan en el ámbito escolar; así, apreciamos cómo la media más alta es la relacionada con el dominio escuela $(\overline{\mathrm{X}}=3,57 ; \mathrm{CV}=47,90)$. Por lo general, los alumnos de EP no suelen asistir a eventos relacionados con la lectura o la escritura, aunque la Feria del Libro es el evento que obtiene mejores resultados $(\overline{\mathrm{X}}=3,15 ; \mathrm{CV}=63,69)$.

Figura 2. Dimensión Producción y consumo cultural

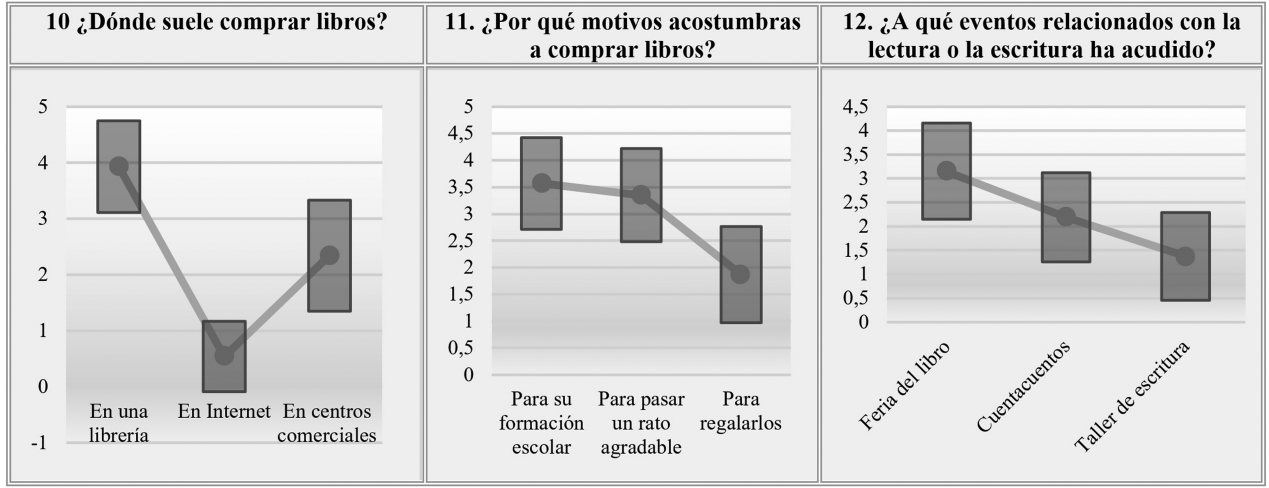

Fuente: Elaboración propia 
En consonancia con las apreciaciones anteriores, el análisis de la dimensión Cultura de la memoria reitera el escaso número de eventos letrados que se desarrollan en las bibliotecas públicas y escolares. Las medias desvelan que los estudiantes de EP no hacen uso de las bibliotecas y que cuando las usan, desempeñan la función de sala de lectura $(\overline{\mathrm{X}}=2,78$; $\mathrm{CV}=73,02)$ o servicio de préstamo $(\overline{\mathrm{X}}=2,64 ; \mathrm{CV}=76,52)$; sin embargo, en ambos usos de la biblioteca se observa una importante dispersión de las opiniones de los estudiantes respecto al valor promedio de las respuestas.

\section{Figura 3. Dimensión Cultura de la memoria}

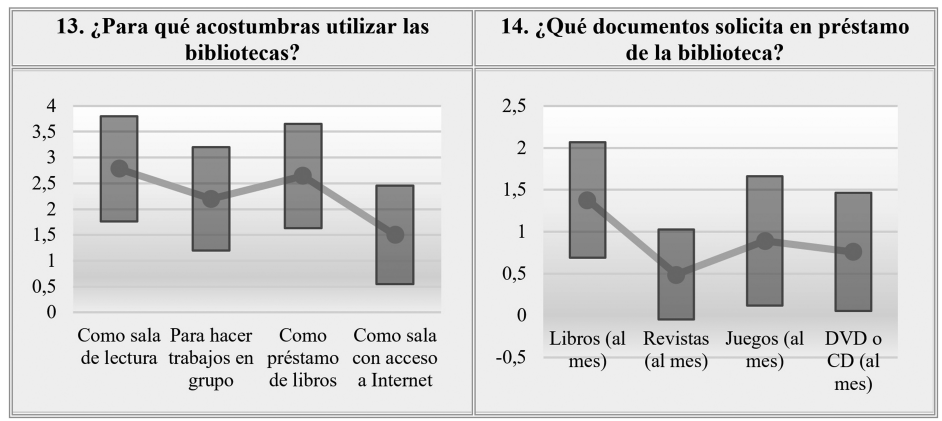

Fuente: Elaboración propia

Los resultados de la dimensión Cultura de la instrucción subrayan la dependencia del libro de texto como recurso más frecuente en el dominio escuela $(\overline{\mathrm{X}}=4,05 ; \mathrm{CV}=34,81)$. En consecuencia, encontramos un escaso uso de otro tipo de lecturas en su formación, como artículos de revista $(\overline{\mathrm{X}}=1,12 ; \mathrm{CV}=133,93)$ o publicaciones de Internet $(\overline{\mathrm{X}}=1,94 ; \mathrm{CV}=98,97)$. Los docentes no suelen fomentar la creación de debates en relación a las lecturas realizadas en el aula; de hecho, las medias más altas las alcanzan los ítems relacionados con la realización de resúmenes $(\bar{x}=3,53 ; C V=46,18)$ y preguntas sobre lo leído $(\bar{x}=3,93 ; C V=38,17)$. Ante las tareas planteadas por el profesor, los escolares coindicen en realizar dos acciones principales: búsqueda de información $(\overline{\mathrm{X}}=3,53 ; \mathrm{CV}=46,46)$ y revisión de la escritura $(\overline{\mathrm{X}}=3,72$; $\mathrm{CV}=43,01)$.

Figura 4. Dimensión Cultura de la instrucción

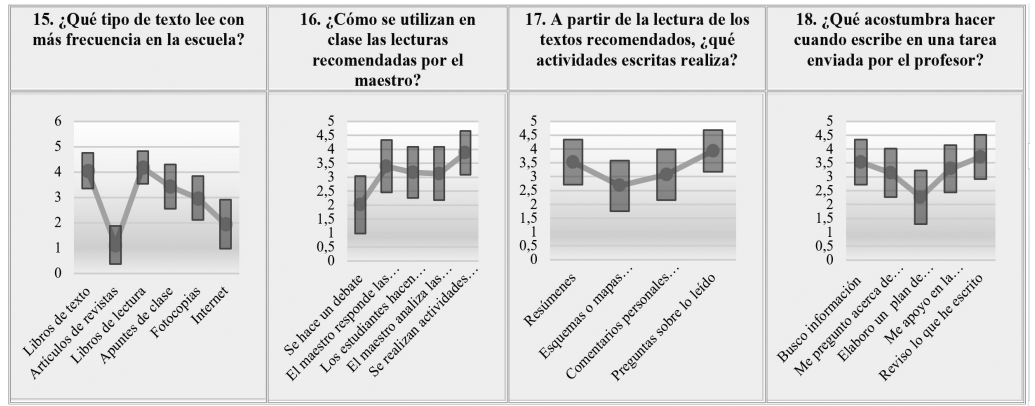

Fuente: Elaboración propia 


\subsection{Perfiles alfabetizadores de los alumnos}

\subsubsection{Alfabetización Personal}

El análisis de clúster bietápico realizado sobre las prácticas letradas relacionadas con la dimensión Alfabetización personal ha permitido clasificar las respuestas de 1213 alumnos de la muestra $(78,8 \%)$ en 2 perfiles diferentes, siendo el clúster 2 el que aglutina más sujetos $(40,9 \%)$, y el clúster 1 , el que menos $(37,9 \%)$.

Tabla 5. Distribución de los clúster correspondientes a la Alfabetización personal

\begin{tabular}{|c|c|c|c|c|}
\hline \multicolumn{5}{|c|}{ Distribución del clúster } \\
\hline \multirow{3}{*}{ Clúster } & N & $\%$ de combinados & $\%$ del Total \\
\cline { 2 - 5 } & 1 & 583 & $48,1 \%$ & $37,9 \%$ \\
\cline { 2 - 3 } & 2 & 630 & $51,9 \%$ & $40,9 \%$ \\
\cline { 2 - 3 } & Combinado & 1213 & & $78,8 \%$ \\
\cline { 2 - 3 } Casos excluidos & 327 & \multirow{2}{*}{$100,0 \%$} & $21,2 \%$ \\
\cline { 3 - 3 } & 1540 & & $100,0 \%$ \\
\hline \multicolumn{2}{|c|}{ Total } & &
\end{tabular}

Fuente: Elaboración propia

De una parte, el clúster 1 representa a alumnos de 8 y 9 años que cursan $3^{\circ}$ o $4^{\circ}$ de EP y que están matriculados en centros con un ISC alto, como es el caso del Centro 2, o centros que tienen ISC medio (como el Centro 7). Este perfil subraya una amplia variedad de eventos letrados en dominios diversos que relacionan la lectura con la finalidad de aprender, pasar un rato agradable o buscar información. Estos escolares presentan hábitos menos definidos hacia la escritura, que circunscriben exclusivamente a la escuela $(\overline{\mathrm{X}}=4,34)$ y con predominio de la lectura impresa $(\overline{\mathrm{X}}=4,52)$. De otra parte, el clúster 2 describe un perfil que tiene una menor motivación hacia la lectura. Sus preferencias lectoras tienden a una lectura en la red, ya sean blogs o páginas web. Estos estudiantes tienen entre 11 y 13 años, cursan $6^{\circ}$ de EP y están matriculados en centros con ISC bajo (véase Tabla 6). Este perfil identifica a alumnos con un mayor desarrollo de la competencia digital, obteniendo puntuaciones más altas en el uso de redes sociales $(\overline{\mathrm{X}}=1,97)$, foros $(\overline{\mathrm{X}}=1,63) \mathrm{y}$, sobre todo, en el uso de WhatsApp $(\overline{\mathrm{X}}=3,88)$. 
Celia Moreno, Fernando Guzmán y Eduardo García $\quad$ Los Hábitos de Lectura y Escritura en los...

Tabla 6. Perfiles de Alfabetización personal del alumnado considerando sus respuestas al Autoinforme, su edad, sexo, curso y centro educativo

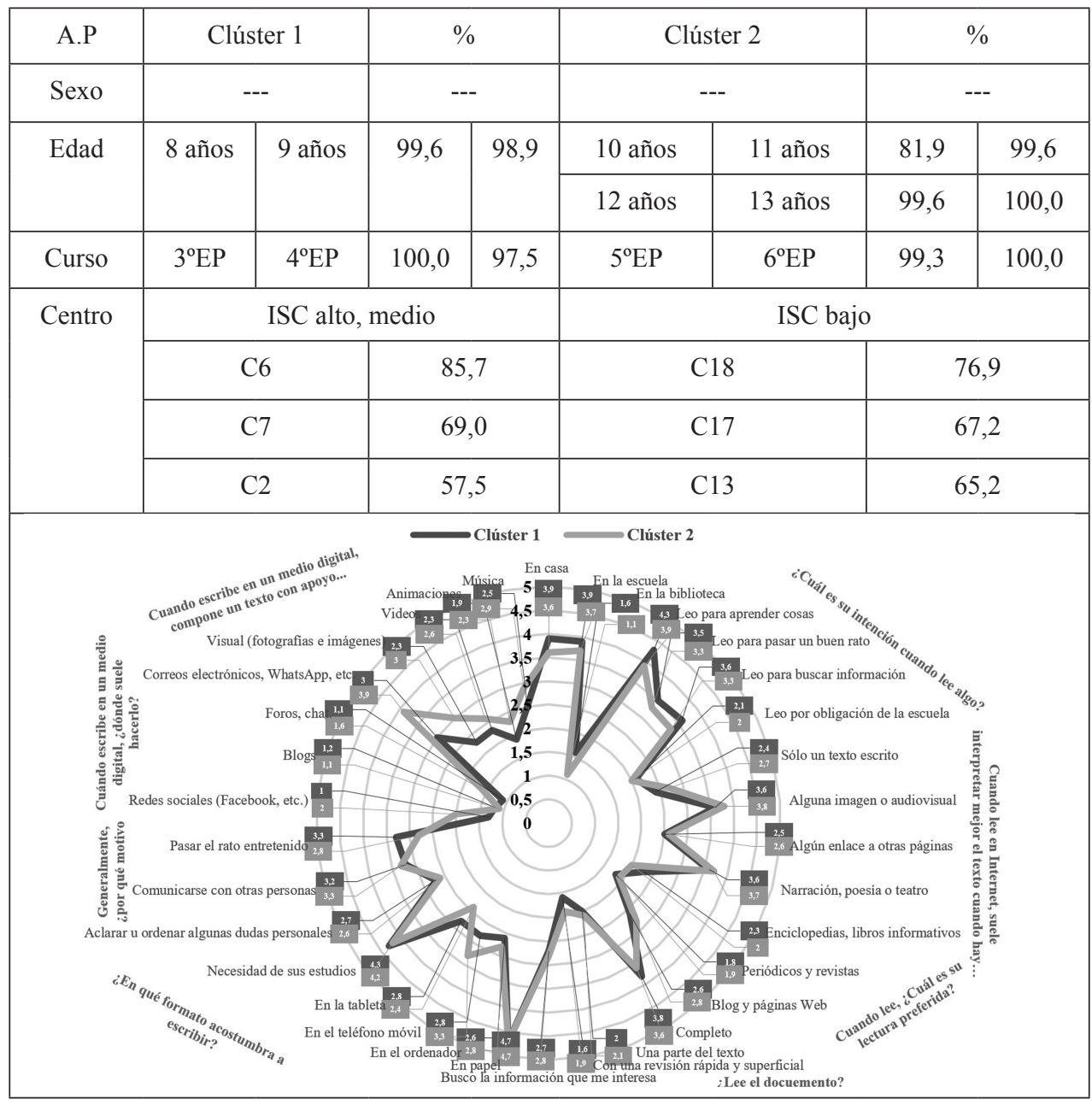

Fuente: Elaboración propia

\subsubsection{Producción y consumo cultural}

El análisis de clúster realizado sobre los eventos letrados relacionados con el mercado editorial (Producción y Consumo Cultural) ha permitido clasificar las respuestas de 1386 alumnos $(89,9 \%)$ en 4 perfiles diferentes, siendo el primer perfil (clúster 1) donde más sujetos se incluyen y el segundo, el que menos $(19,2 \%)$. 
Tabla 7. Distribución de los clúster correspondientes a la dimensión Producción y consumo cultural

\begin{tabular}{|c|c|c|c|c|}
\hline \multicolumn{5}{|c|}{ Distribución del clúster } \\
\hline \multirow{3}{*}{ Clúster } & 1 & $\mathrm{~N}$ & $\%$ de combinados & $\%$ del Total \\
\cline { 2 - 5 } & 2 & 379 & 27,4 & 24,6 \\
\cline { 2 - 5 } & 3 & 341 & 21,4 & 19,2 \\
\cline { 2 - 4 } & 4 & 368 & 24,6 & 22,1 \\
\cline { 2 - 3 } & Combinado & 1384 & 26,6 & 23,9 \\
\cline { 2 - 3 } & \multicolumn{3}{|c|}{100,0} & 89,9 \\
\hline \multicolumn{2}{|c|}{ Casos excluidos } & 156 & & 10,1 \\
\hline \multicolumn{2}{|c|}{ Total } & 1540 & & 100,0 \\
\hline
\end{tabular}

Fuente: Elaboración propia

Los diferentes clústeres que han surgido en el análisis de la dimensión de Producción y consumo cultural nos llevan a identificar 4 perfiles, dos de ellos con rasgos bien diferenciados (clúster 1 versus clúster 2). Así, el clúster 1 reproduce un perfil de alumno de 8 años de edad, que cursa $3^{\circ}$ de E.P y que se encuentra matriculado en centros de ISC medio o alto. Estos escolares consumen un mayor número de productos alfabetizadores que los representados en el clúster 4. Así, compran libros en librerías e Internet como respuesta a una necesidad de su formación escolar o interés personal. Además, asisten a eventos relacionados con la lectura y la escritura (Feria del libro, cuentacuentos, etc.). Por contra, el clúster 2 representa un perfil de alumnos matriculados en centros de ISC medio o bajo que no suele comprar libros de forma habitual y no suelen asistir a eventos relacionados con la lectura y la escritura. Cuando adquieren libros, lo hacen por exigencia de su formación escolar $(\overline{\mathrm{X}}=3,43)$ y, principalmente, en librerías $(\overline{\mathrm{X}}=3,98)$.

Tabla 8. Perfiles de Producción y Consumo Cultural del alumnado considerando sus respuestas al Autoinforme, su edad, sexo, curso y centro educativo

\begin{tabular}{|c|c|c|c|c|c|c|c|c|}
\hline P.C.C & Clúster 1 & $\%$ & Clúster 2 & $\%$ & Clúster 3 & $\%$ & Clúster 4 & $\%$ \\
\hline Sexo & --- & --- & --- & --- & --- & --- & --- & --- \\
\hline \multirow[t]{3}{*}{ Edad } & \multirow[t]{3}{*}{8 años } & \multirow[t]{3}{*}{99,7} & \multirow[t]{3}{*}{9 años } & \multirow[t]{3}{*}{73,8} & \multirow[t]{3}{*}{10 años } & \multirow[t]{3}{*}{82,7} & 11 años & 78,2 \\
\hline & & & & & & & 12 años & 92,5 \\
\hline & & & & & & & 13 años & 100,0 \\
\hline Curso & $3^{\circ} \mathrm{EP}$ & 98,3 & $4^{\circ} \mathrm{EP}$ & 91,5 & $5^{\circ} \mathrm{EP}$ & 98,8 & $6^{\circ} \mathrm{EP}$ & 100,0 \\
\hline \multirow[t]{4}{*}{ Centro } & \multicolumn{2}{|c|}{ ISC medio, alto } & \multicolumn{2}{|c|}{ ISC medio, bajo } & \multicolumn{2}{|c|}{---} & \multicolumn{2}{|c|}{ ISC bajo } \\
\hline & C6 & 50,0 & C9 & 28,8 & $\mathrm{C} 3$ & 28,2 & $\mathrm{C} 18$ & 50,0 \\
\hline & $\mathrm{C} 7$ & 41,7 & $\mathrm{C} 16$ & 27,2 & $\mathrm{C} 15$ & 29,3 & $\mathrm{C} 14$ & 37,3 \\
\hline & $\mathrm{C} 1$ & 32,8 & $\mathrm{C} 4$ & 27,2 & C11 & 34,0 & $\mathrm{C} 17$ & 35,7 \\
\hline
\end{tabular}


Celia Moreno, Fernando Guzmán y Eduardo García $\quad$ Los Hábitos de Lectura y Escritura en los...

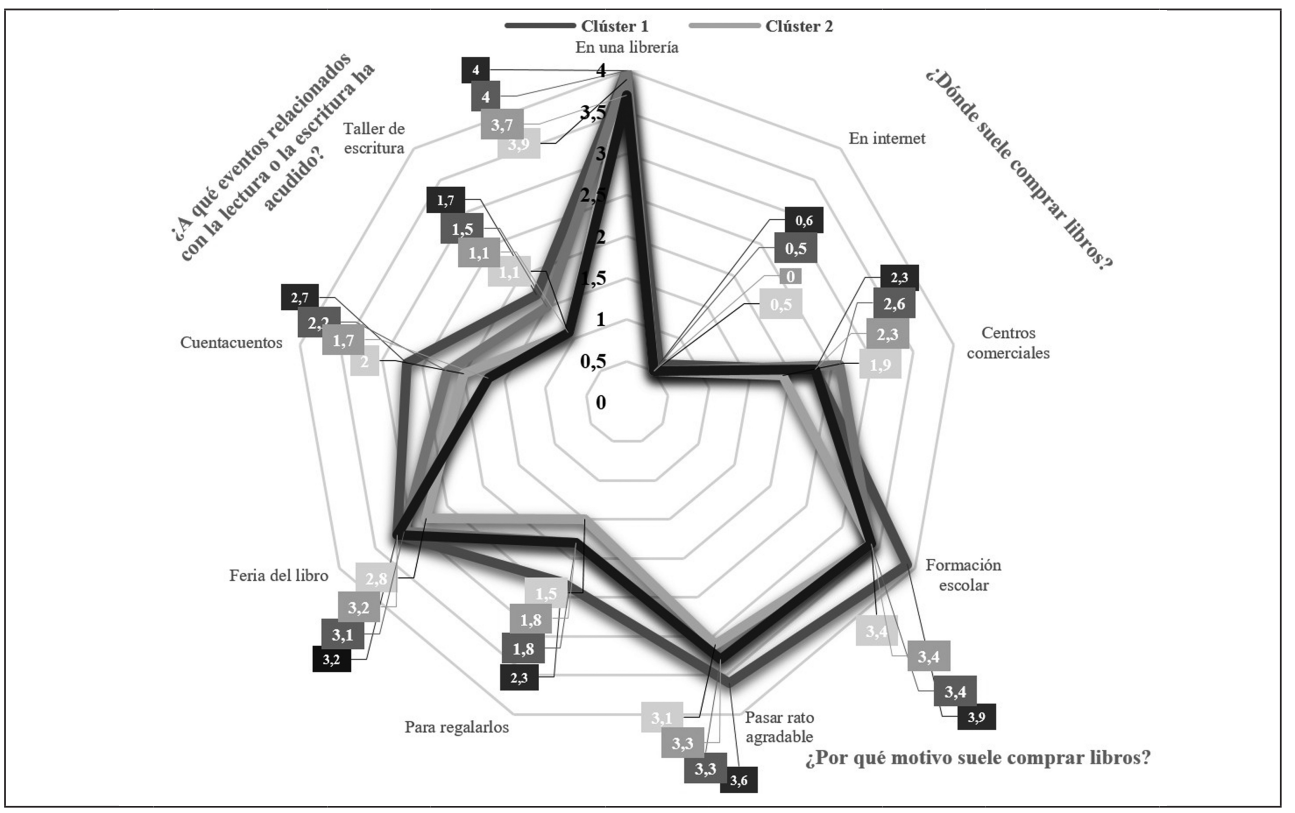

Fuente: Elaboración propia

\subsubsection{Cultura de la memoria}

En relación a la dimensión Cultura de la memoria (Tabla 9), el análisis de clúster bietápico ha permitido clasificar las respuestas de los escolares en 2 perfiles diferentes. El $91,4 \%$ de los alumnos ha quedado ubicado en uno de estos dos clústeres, donde el segundo perfil (clúster 2) incluye más sujetos $(46,6 \%)$ que el primero $(44,9 \%)$.

Tabla 9. Distribución de los clúster correspondientes a la dimensión Cultura de la memoria

\begin{tabular}{|c|l|c|c|c|}
\hline \multicolumn{5}{|c|}{ Distribución del clúster } \\
\hline \multirow{2}{*}{ Clúster } & 1 & N & \% de combinados & $\%$ del Total \\
\cline { 2 - 3 } & 2 & 691 & 49,1 & 44,9 \\
\cline { 2 - 3 } & Combinado & 1408 & 50,9 & 46,6 \\
\hline \multicolumn{2}{|l|}{ Casos excluidos } & 132 & 100,0 & 91,4 \\
\hline \multicolumn{2}{|l|}{ Total } & 1540 & & 8,6 \\
\cline { 2 - 3 } & & & 100,0 \\
\hline
\end{tabular}

Fuente: Elaboración propia 
La dimensión Cultura de la memoria no es una dimensión que facilite la construcción de distintos perfiles alfabetizadores. Los diferentes análisis realizados evidencian un escaso uso de las bibliotecas públicas o escolares. La variable ISC ayuda a identificar particularidades en los comportamientos de los estudiantes. Así, los escolares clasificados en el clúster 2 cursan $5^{\circ}$ o $6^{\circ}$ de E.P., están matriculados en centros con ISC medio o medio-bajo y hacen un mayor uso de los recursos ofrecidos en las bibliotecas (Tabla 10).

Tabla 10. Perfiles de Cultura de la memoria del alumnado considerando sus respuestas al Autoinforme, su edad, sexo, curso y centro educativo

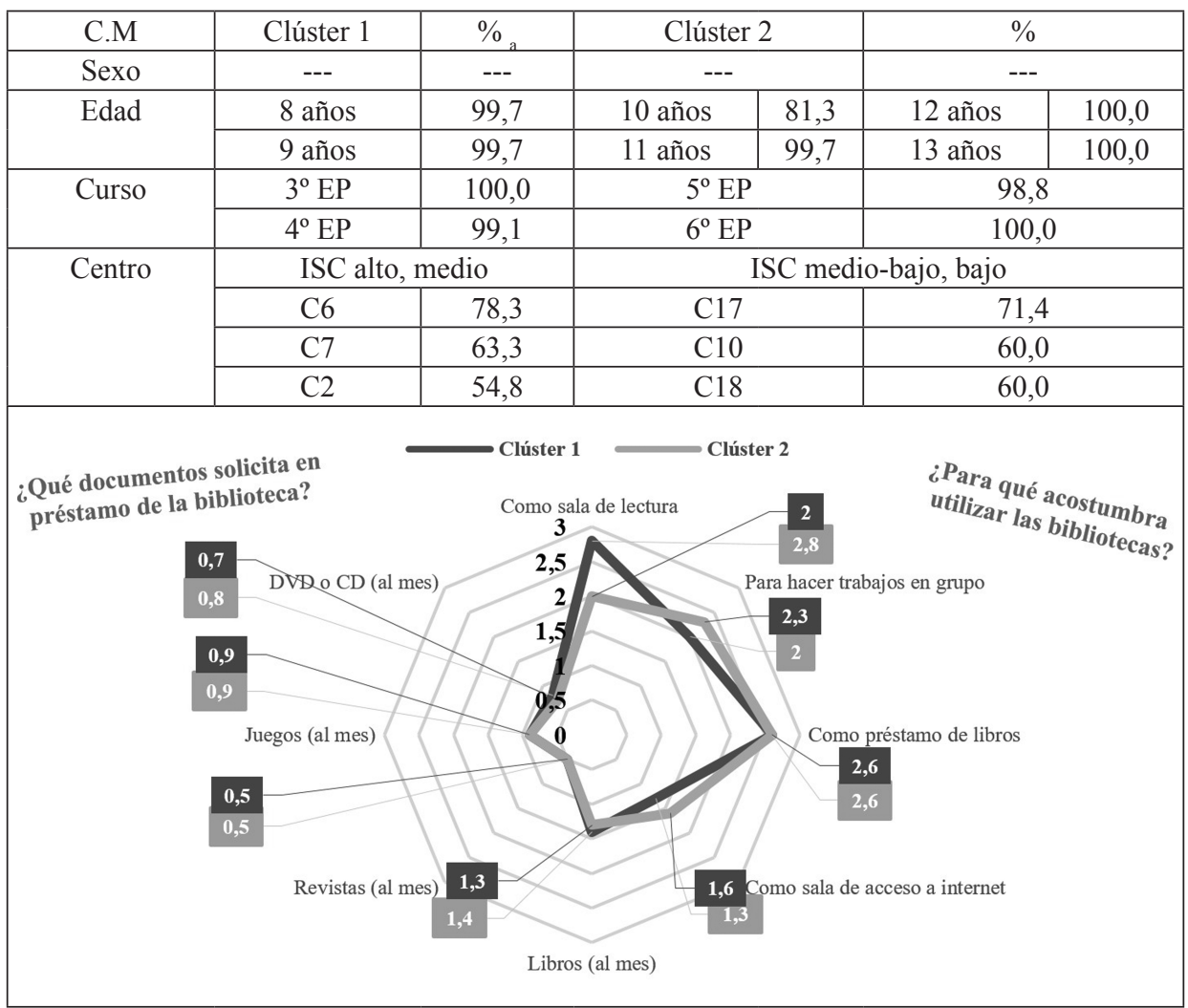

Fuente: Elaboración propia

\subsubsection{Cultura de la instrucción}

El análisis de clúster bietápico ha permitido clasificar las respuestas de los alumnos al Autoinforme relacionadas con la Cultura de la instrucción en tres perfiles distintos. Así, el $82,9 \%$ de los alumnos ha quedado ubicado en uno de los tres perfiles identificados, siendo el clúster 1 el que más sujetos incluye (32,9\%), y el tercero, el que menos $(22 \%)$. 
Tabla 11. Distribución de los clúster correspondientes a la dimensión Cultura de la Instrucción

\begin{tabular}{|c|c|c|c|c|}
\hline \multicolumn{5}{|c|}{ Distribución del clúster } \\
\hline & & $\mathrm{N}$ & $\%$ de combinados & $\%$ del Total \\
\hline \multirow[t]{4}{*}{ Clúster } & 1 & 506 & 32,9 & 32,9 \\
\hline & 2 & 432 & 28,1 & 28,1 \\
\hline & 3 & 339 & 22,0 & 22,0 \\
\hline & Combinado & 1277 & 82,9 & 82,9 \\
\hline \multicolumn{2}{|c|}{ Casos excluidos } & 263 & & 17,1 \\
\hline \multicolumn{2}{|c|}{ Total } & 1540 & & 100,0 \\
\hline
\end{tabular}

Fuente: Elaboración propia

El estudio de esta dimensión permite conocer el tipo de actividades relacionadas con la lectura y la escritura que prevalecen en la escuela. El ISC del centro no introduce variaciones en los perfiles descritos, por lo que las diferencias vienen únicamente marcadas por el curso y edad de los escolares. El análisis realizado identifica dos perfiles con características opuestas, refiriéndonos a los clústeres 1 y 2 . Los alumnos agrupados en el clúster 1 destacan por la diversidad de textos utilizados en la escuela y la variedad de actividades realizadas (debates $[\bar{X}=3,02]$, resúmenes $[\bar{X}=3,95]$, reflexiones $[\bar{X}=4,20]$, etc.). Estos muestran más estrategias de autorregulación ante las tareas demandadas. En el polo opuesto, el clúster 2 describe un perfil escolar que promueve el uso casi exclusivo del libro de texto $(\overline{\mathrm{X}}=3,40)$ y/o el libro de lectura $(\overline{\mathrm{X}}=3,67)$. En este sentido, destaca la realización de actividades escritas sobre la lectura $(\bar{X}=3,03)$, esquemas o mapas conceptuales $(\bar{X}=4,59)$. Son alumnos que poseen escasas estrategias para organizar y gestionar la información y las tareas que realizan son una mera repetición del conocimiento aprendido.

Tabla 12. Perfiles de Cultura de la instrucción del alumnado considerando sus respuestas al Autoinforme, su edad, sexo, curso y centro educativo

\begin{tabular}{|c|c|c|c|c|c|c|}
\hline C.I & Clúster 1 & $\%$ & Clúster 2 & $\%$ & Clúster 3 & $\%$ \\
\hline Sexo & --- & --- & --- & --- & --- & --- \\
\hline \multirow[t]{3}{*}{ Edad } & \multirow[t]{3}{*}{8 años } & \multirow[t]{3}{*}{75,0} & 9 años & 54,9 & 11 años & 77,1 \\
\hline & & & \multirow[t]{2}{*}{10 años } & \multirow[t]{2}{*}{53,0} & 12 años & 95,4 \\
\hline & & & & & 13 años & 100,0 \\
\hline \multirow[t]{2}{*}{ Curso } & \multirow[t]{2}{*}{$3^{\circ} \mathrm{EP}$} & \multirow[t]{2}{*}{73,6} & $4^{\circ} \mathrm{EP}$ & 59,8 & \multirow[t]{2}{*}{$6^{\circ} \mathrm{EP}$} & \multirow[t]{2}{*}{95,9} \\
\hline & & & $5^{\circ} \mathrm{EP}$ & 50,8 & & \\
\hline \multirow[t]{4}{*}{ Centro } & \multicolumn{2}{|c|}{---} & \multicolumn{2}{|c|}{---} & \multicolumn{2}{|c|}{---} \\
\hline & $\mathrm{C} 16$ & 60,0 & $\mathrm{C} 6$ & 89,5 & \multirow[t]{3}{*}{$\mathrm{C} 18$} & \multirow[t]{3}{*}{57,1} \\
\hline & $\mathrm{C} 10$ & 58,8 & $\mathrm{C} 17$ & 43,0 & & \\
\hline & $\mathrm{C} 1$ & 48,4 & $\mathrm{C} 2$ & 42,9 & & \\
\hline
\end{tabular}




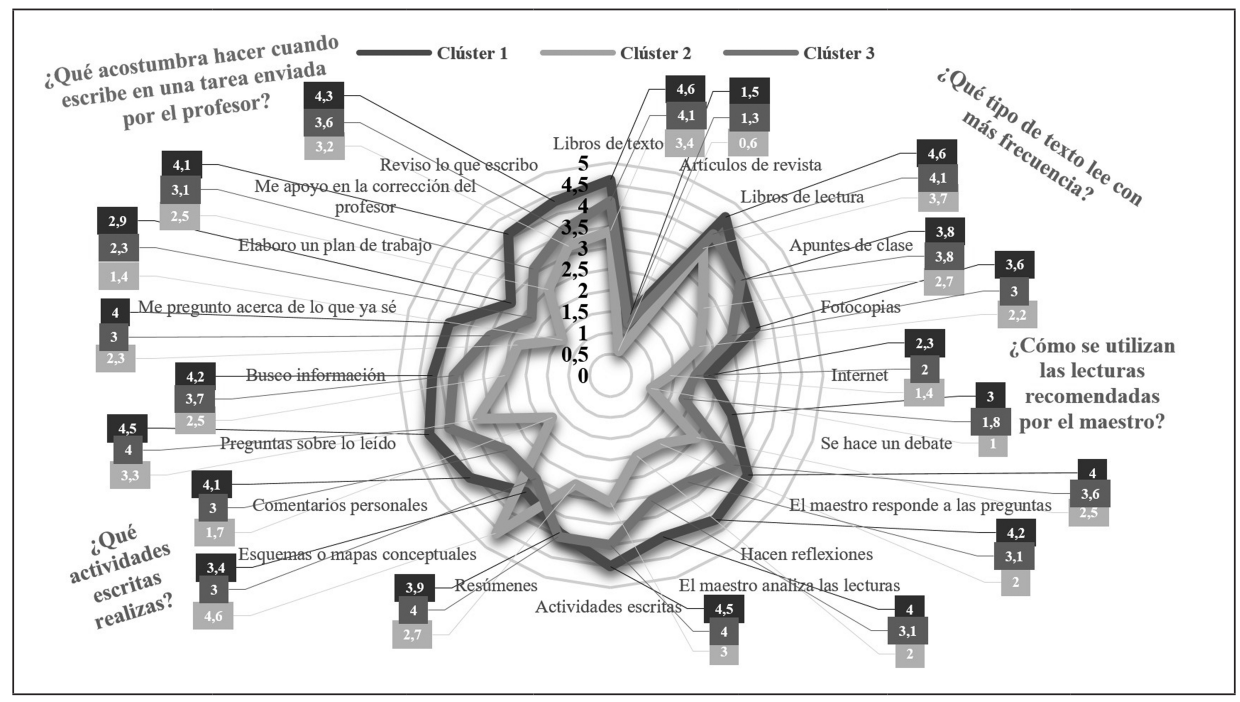

Fuente: Elaboración propia

\section{Discusión}

La alfabetización en EP es el resultado de la interacción entre eventos letrados originados en la escuela, la familia o la comunidad de amigos. Estos eventos están mediatizados por la influencia que ejerce el ISC de los centros y por las diferencias individuales de los alumnos (Neuman \& Celano, 2001). Los resultados de esta investigación indican que los factores sociales que interactúan en la alfabetización infantil poseen una mayor relevancia que las diferencias individuales. Estos factores sociales hacen referencia a variables socioeconómicas, medidas a través del ISE, que tienen una influencia asimétrica sobre la alfabetización familiar, escolar o propia en otras comunidades discursivas (Saint-Laurent \& Giasson, 2005). Así, los alumnos que estudian en centros con ISC alto muestran una mayor permeabilidad a las prácticas alfabetizadoras desarrolladas en el dominio escuela. Estas prácticas formales determinan en buena medida las prácticas personales y sociales de alfabetización (aprendizajes no formales e informales). En cambio, los alumnos que estudian en centros con ISC bajo desarrollan una menor dependencia de las prácticas escolares. Las prácticas alfabetizadoras de estos alumnos son de carácter vernáculo y se desarrollan en el dominio familiar, en el barrio o en su comunidad de amigos.

Las diferencias halladas en nuestro estudio confirman que la influencia del dominio escuela está vinculada a los valores culturales asociados a la alfabetización desarrollada por las familias (Dunsmore \& Fisher, 2010). Aquellos escolares cuyas familias tienen valores culturales próximos a los definidos por la escuela construyen una identidad basada en unas prácticas alfabetizadoras formales. Por el contrario, aquellos otros escolares, cuyas familias se sienten menos identificados con los valores alfabetizadores de la escuela, presentan una identidad influenciada por otras comunidades discursivas, como el barrio o el hogar. 
El proceso alfabetizador actual se caracteriza por utilizar diversas fuentes procedentes de distintos dominios, medios y agentes. Los alumnos con ISC bajo escriben y leen utilizando una diversidad de medios impresos y digitales dentro y fuera de la escuela. En cambio, los alumnos con ISC Alto muestran una alfabetización más homogénea, donde los aprendizajes formales se desarrollan a través de medios impresos en el dominio escuela. En consecuencia, la heterogeneidad de las identidades del alumnado está construida a partir del conjunto de comunidades discursivas y valores sociales con los que interacciona habitualmente.

Actualmente, la escuela comparte con otros dominios el papel social de la alfabetización (Davies \& Merchant, 2009). Los eventos alfabetizadores descritos en nuestra investigación dibujan una escuela cuya alfabetización es ajena a los nuevos focos alfabetizadores como la cultura popular o las TIC (Williams, 2009). Los resultados de esta investigación muestran que las prácticas alfabetizadoras en la Cultura de la instrucción resultan homogéneas entre los centros. Los eventos letrados promovidos por las escuelas ofrecen una respuesta análoga a necesidades educativas muy distintas. En consecuencia, la cultura escolar no resulta un elemento diferenciador ni compensatorio en el desarrollo de la alfabetización en EP (Gil, 2009).

El mantenimiento por parte de la escuela del liderazgo en la sociedad actual requiere de la creación de un tercer espacio alfabetizador, donde convivan diferentes alfabetizaciones surgidas en dominios distintos a la escuela (Moje, Ciechanowsky, Kramer, et al., 2004). La incorporación de las experiencias alfabetizadoras vernáculas de los alumnos permitiría aprovechar nuevas competencias comunicativas para la lectura y escritura de discursos multimodales (Levy, 2008) y el desarrollo de una alfabetización híbrida (Chouliaraki \& Fairclough, 2007).

La distancia existente entre la alfabetización escolar y vernácula explica las diferencias encontradas en nuestro estudio asociadas a la edad de los estudiantes. Esta investigación señala que los eventos alfabetizadores de los escolares de 8 y 9 años mantienen cierto equilibrio entre lo vernáculo y lo escolar. Posteriormente, los escolares de 11 años trasladan el foco de interés de sus eventos alfabetizadores hacia una alfabetización vernácula a través de las TIC.

Nuestro estudio dibuja un proceso de alfabetización escolar en EP que permanece al margen de las necesidades alfabetizadoras cotidianas de los niños. Esta alfabetización no ha dado respuesta a los retos del desarrollo de la lectura y la escritura en el siglo XXI. La creación de un tercer espacio alfabetizador, un cambio respecto al valor social de la comunicación vernácula y la incorporación efectiva de la web 2.0 contribuirían a fortalecer el papel alfabetizador de la escuela. La teoría del "tercer espacio" propuesta por Bhabha (1994), Gutiérrez, Baquedano-López, Álvarez y Chiu (1999) y Soja (1996) presenta una hipótesis sobre la creación de un nuevo espacio, tanto físico como social, de alfabetización entre el hogar y la escuela. La experiencia de alfabetización del estudiante es compleja en este tercer espacio, dada la variedad de textos y códigos a los que se enfrentan (Bearne, 2005). La construcción de este tercer espacio puede articularse tomando en consideración el contexto del estudiante, así como la variedad de géneros discursivos dentro y fuera de la escuela (Gutiérrez et al., 2003; Pahl \& Allan, 2011). La incorporación en las aulas de una pedagogía de los géneros (como ha propuesto la Escuela de Sydney) incorporaría una metodología nueva que facilita la creación de ciclos de interacción entre los estudiantes, los profesores y sus familias (Rose \& Martin, 2012) e integraría un carácter comunicativo y funcional de la lectura y la escritura multimodales en el aula (Rowsell, Kress, Pahl, \& Street, 2013). En conclusión, el desarrollo del tercer espacio en la escuela se convertiría en un puente entre el curriculum escolar de EP, el conocimiento que los niños adquieren en su 
vida cotidiana y el acceso de los estudiantes a las prácticas más complejas de alfabetización en la escuela (Moje et al., 2004).

\section{REFERENCES}

Barton, D., \& Hamilton, M. (1998). Local literacies. Reading and writing in one community. London: Routledge.

Barton, D., \& Lee, C.K.M. (2012). "Redefining vernacular literacies in the age of Web 2.0", in Applied Linguistics, 33, 3: 282-298.

Barton, D. \& Papen, U. (eds.) (2010). The Anthropology of Writing: Understanding textually mediated worlds. London, GB: Continuum.

Bearne, E. (2005). Multimodal texts: What they are and how children use them. In J. Evans (Ed.), Literacy moves on: Using popular culture, new technologies and critical literacy in the primary classroom (pp. 13-29). London: David Fulton.

Bhabha, H.K. (1994). The location of culture. New York: Routledge.

Bourdieu, P. \& Passeron, J. C. (1990). Reproduction in education, society and culture. London: Sage.

Brandt, D., \& Clinton, K. (2002). "Limits of the Local: Expanding Perspectives on Literacy as a Social Practice", in Journal of Literacy Research, 34, 3: 337-356.

Caro, D. H., McDonald, J. T., \& Willms, J. D. (2009). "Socio economic status and academic achievement trajectories from childhood to adolescence" in Canadian Journal of Education, 32, 3: 558-590.

CIS (2016). "Barómetro de febrero 2016". Madrid: Centro de Investigaciones Sociológicas. Available from: http://www.cis.es, accessed 20 April, 2016.

CIS (2016). Barómetro de septiembre 2016. Madrid: Centro de Investigaciones Sociológicas. Available from: http://www.cis.es accessed 20 April, 2016.

Compton-Lilly, C. (2009). "Listening to families over time: Seven lessons learned about literacy in families", in Language Arts, 86, 6: 449-457.

Compton-Lilly, C. \& Green, S. (eds.) (2011). Bedtimes stories and book reports: connecting parent involvement and family literacy. New York: Teachers College Press.

Cope, B. \& Kalantzis, M. (2009). “"Multiliteracies': New literacies, new learning”, in Pedagogies. An International Journal, 4, 3: 164-195.

Corral Blanco, N., Zurbano Fernández, E., Blanco Fernández, A., García Honrado, I., \& Ramos Guajardo, A.B. (2013). "Estructura del entorno educativo familiar: su influencia sobre el rendimiento y el rendimiento diferencial" in En PIRLS-TIMSS 2011. Estudio Internacional de progreso en la comprensión lectora, matemática y ciencias. Madrid: Secretaría General Técnica, 9-32.

Chouliaraki, L. \& Fairclough, N. (2007). Discourse in Late Modernity. Rethinking Critical Discourse Analysis. Edinburgh: Edinburgh University Press.

Dagenais, D., Toohey, K., Fox, A.B., \& Singh, A. (2016). "Multilingual and multimodal composition at school: ScribJab in Action", in Language and Education, 31, 3: 263-282.

Davies, J. \& Merchant, G. (2009). Web 2.0 for Schools. Learning and Social Participation. New York: Peter Lang.

Desert, M., Preaux, M., \& Jund, R. (2009). "So young and already victims of stereotype threat: Socio-economic status and performance of 6 to 9 years old children on raven's progressive matrices" in European Journal of Psychology of Education, 24, 2:207-218.

Dixon, L.Q. \& Wu, S. (2014). "Home language and literacy practices among immigrant second- 
language learners", in Language Teaching, 47, 4: 414-449.

Dunsmore, K. \& Fisher, D. (eds.) (2010). Bringing literacy home. Newark, DE: International Reading Association.

Duursma, E., Meijer, A., \& De Bot, K. (2017). "The impact of home literacy and family factor son screen media use among Dutch preteens", in Journal of Child and Family Studies, 26, 2: 612-622.

Formby, S. (2014). Children's early literacy practices at home and in early years settings: Second annual survey of parents and practitioners. London: National Literacy Trust.

Gee, J.P. (1999). "Reading and the new literacy studies: Reframing the national academy of sciences report on reading" in Journal of Literacy Research, 31, 3: 355-374.

Gil Flores, J. (2009). "Hábitos y actitudes de las familias hacia la lectura y competencias básicas del alumnado", in Revista de Educación, 350: 301-322.

Gil Flores, J. (2013). "Medición del nivel socioeconómico familiar en el alumnado de Educación Primaria", in Revista de Educación, 362: 298-322.

González, L. (2017). "Hábitos lectores y políticas habituales de lectura”, in J.A. Millán (Cood.), La lectura en España. Informe 2017. Madrid: Federación de Gremios de Editores de España, 81-91.

Gómez Soto, I. (1999). Mito y realidad de la lectura. Los hábitos lectores en la España actual. Madrid: Endymion.

Goodfellow, R. (2011). "Literacy, literacies and the digital in higher education", in Teaching in Higher Education, 16, 1: 131-144.

Gregory, E. \& Williams, A. (2000). "Work or play? Unofficial literacies in the lives of two East London Communities", in M. Martin-Jones \& K. Jones (eds.), Multilingual literacies: Reading and writing different world. Amsterdam: John Benjamins, 37-54.

Gutiérrez, K. D., Baquedano-López, P., \& Tejeda, C. (2003). "Rethinking diversity: Hybridity and hybrid language practices in the third space", in Mind, Culture and Activity, 6:4, 286-303.

Hashemi, S.S. (2017). Socio-semiotic patterns in digital meaning-making: semiotic choice as indicator of communicative experience. Language and Education, 31(5), 432-448.

Heath, S.B. (1983). Ways with Words: Language, Life, and Work in Communities and Classrooms. Mass: Cambridge University Press.

Hull, G. \& Schultz, K. (2002). "Connecting schools with Out-of-School worlds. Insights from recent research on literacy in non-school settings", in G. Hull \& K. Schultz (Eds.), School's Out! Bridging Out-of-School Literacies with Classroom Practice. New York and London: Teachers College Press, 32-57.

Instituto Nacional de Evaluación Educativa (2013). PIRLS-TIMSS 2011. Estudio Internacional de progreso en comprensión lectora, matemáticas y ciencias. Vol. I y II. Madrid: Ministerio de Educación, Cultura y Deporte.

Kalantzis, M., \& Cope, B. (2000). "Changing the role of schools", in B. Cope \& M. Kalantzis Multiliteracies. Literacy learning and the design of social futures London: Routledge, 116-144.

Kress, G. (2003). Literacy in the New Media Age. London: Routledge.

Lancaster, L. (2003). "Beginning at the beginning: How a young child constructs time multimodality", in C. Jewitt \& G. Kress (Eds.), Multimodal literacy New York: Peter Lang, 107-122.

Levy, R. (2008). “"Third spaces' are interesting places: Applying 'third space theory' to nurseryaged children's constructions of themselves as readers", in Journal of Early Childhood Literacy, 8, 1: 43-66.

Lillis, T. (2013). The sociolinguistics of writing. Edinburgh: Edinburgh University Press. 
Lotherington, H. (2017). "Elementary Language Education in Digital Multimodal and Multiliteracy Contexts", in Language in Education and Technology, 1-15.

Maybin, J. (2006). Children's voices. Talk, knowledge and identity. Basingstoke: Palgrave Macmillan.

MEC (2015). Encuesta de hábitos y prácticas culturales 2014-2015. Madrid: Secretaría General Técnica (Subdirección General de Estadística y Estudios).

Millán, J.A. (Coor.) (2017). La lectura en España. Informe 2017. Madrid: Federación de Gremios de Editores de España.

Mocker, D.W. \& Spear, G.E. (1982). Lifelong learning: formal, nonformal, informal, and selfdirected. Columbus, Ohio: The Ohio State University.

Moje, E.B., Ciechanowski, K.M., Kramer, K., Ellis, L., Carrillo, R., \& Collazo, T. (2004). "Working toward third space in content area literacy: An examination of everyday funds of knowledge and discourse", in Reading Research Quarterly, 39, 1: 38-70.

Neuman, D.B. \& Celano, D. (2001). "Access to print in low-income and middle-income communities: An ecological study of four neighbourhoods", in Reading Research Quarterly, 36, 1: 8-26.

New London Group (1996). "A pedagogy of multiliteracies: Designing social futures", in Harvard Educational Review, 66, 1: 60-93.

Pahl, K. (2007). "Creativity in events and practices: a lens for understanding children's multimodal texts", in Literacy, 41, 2: 86-92.

Pahl, K. \& Allan, Ch. (2011). "I don't know what literacy is: Uncovering hidden literacies in a community library using ecological and participatory research methodologies with children", in Journal of Early Childhood Literacy, 11, 2: 190-213.

Pahl, K., \& Rowsell, J. (2012). Literacy and Education. Understanding the New Literacy Studies in the Classroom. London: Sage.

Purcell-Gates, V. (1995). "Literacy at the Harts' and the Larsons': Diversity among poor, innercity families", in The Reading Teacher, 48, 7: 572-578.

Purcell-Gates, V. (1996). "Stories, coupons, and the TV Guide: Relationships between home literacy experiences and emergent literacy knowledge", in Reading Research Quarterly, 31, 4: 406-428.

Rose, D., \& Martin, J. R. (2012). Learning to write, reading to learn: Genre, knowledge and pedagogy in the Sydney School. United Kingdom: Equinox.

Rowsell, J. \& Pahl, K. (2007). "Sedimented identities in texts: Instances of practice. Reading Research Quarterly" 42, 3: 388-404.

Rowsell, J. Kress, G., Pahl, K. and Street B. (2013). "The Social Practice of Multimodal Reading: A New Literacy Studies - Multimodal Perspective on reading", in Alvermann, D.E., Unrau, N.J., \& Ruddell, R.B. (Eds.). (2013). Theoretical models and processes of reading (6th ed.). Newark, DE: International Reading Association, 1182-1207.

S.A. (2013). Hábitos de Lectura y Compra de libros en España 2012. Madrid: Federación de Gremios de Editores de España.

Saint-Laurent, L. \& Giasson, J. (2005). "Effects of a family literacy program adapting parental interventions to first graders' evolution of reading and writing abilities", in Journal of Early Childhood Literacy, 5(3): 253-278.

Soja, E.W. (1996). Thirdspace: Journeys to Los Angeles and other real-and-imagined places. Malden, MA: Blackwell.

Steensel, R. van (2006). "Relations between socio-cultural factors, the home literacy environment and children's literacy development in the first years of primary education", in Journal of Research in Reading, 29, 4: 367-382. 
Celia Moreno, Fernando Guzmán y Eduardo García $\quad$ Los Hábitos de Lectura y Escritura en los...

Street, B.V. (1994). "The New Literacy Studies: Implications for Education and Pedagogy", in Changing English, 1, 1:113-126.

Trujillo Sáez, F. (2017). "El sistema educativo", in J.A. Millán (Cood.), La lectura en España. Informe 2017. Madrid: Federación de Gremios de Editores de España, 97-109.

Tusting, K., \& Barton, D. (2005). "Community-based local literacies Research", in R. Beach, J. Green, M. Kamil, \& T. Shanahan (Eds.), Multidisciplinary perspectives on literacy research. New Jersey: Hampton Press, 243-263.

Van Steensel, R. (2006). "Relations between socio-cultural factors, the home literacy environment, and children's literacy development in the first years of primary education", in Journal of Research in Reading, 29: 367-382.

Williams, B.T. (2009). Shimmering literacies: Popular culture \& reading \& writing online. New York: Peter Lang.

Yin, R. K. (2014). Case Study Research: Design and methods. London: SAGE. 